Enferm Bras 2020;19(4):302-9

https://doi.org/10.33233/eb.v19i4.3992

\title{
ARTIGO ORIGINAL \\ Percepções de enfermeiros sobre as relações interpessoais no cuidado de enfermagem ambulatorial
}

Raquel de Oliveira Martins Fernandes, M.Sc.*, Edith Monteiro de Oliveira*, Anna Karla Nascimento Lima*, Louise Cândido Souza, M.Sc.*, Laércio Deleon de Melo, M.Sc.*, Thelma Spindola, D.Sc. ${ }^{\star *}$, Denise Barbosa de Castro Friedrich, D.Sc. ${ }^{* \star}$

*Enfermeira, Faculdade de Enfermagem da Universidade Federal de Juiz de Fora (FACENFUFJF), Juiz de Fora/MG, ${ }^{\star \star} E n f e r m e i r a$, Professora Associada pela Faculdade de Enfermagem da Universidade do Estado do Rio de Janeiro (UERJ), Rio de Janeiro/RJ, ${ }^{* * *}$ Enfermeira, Professora Convidada pela Faculdade de Enfermagem e Professora Permanente do Programa de Mestrado da Universidade Federal de Juiz de Fora (FACENF-UFJF)

Recebido em 24 de março de 2020; aceito em 21 de agosto de 2020.

Correspondência: Raquel de Oliveira Martins Fernandes, Rua Ministro Amarílio Lopes Salgado, 72/102, 36033-290 Juiz de Fora MG

Raquel de Oliveira Martins Fernandes: raquel.omfernandes@gmail.com,

Denise Barbosa de Castro Friedrich: denisefriedrichenf@gmail.com

Edith Monteiro de Oliveira: enf.editholiveira@outlook.com

Anna Karla Nascimento Lima: annakarla.enf@gmail.com

Louise Cândido Souza: louisecandidosouza@yahoo.com.br

Laércio Deleon de Melo: laerciodl28@hotmail.com

Thelma Spindola: tspindola.uerj@gmail.com

\section{Resumo}

Introdução: As relações interpessoais, por meio da ampliação do diálogo e da promoção da gestão participativa, configuram-se como competência fundamental ao cuidado e ao fortalecimento da humanização na assistência ambulatorial. Objetivo: Compreender as percepções de enfermeiros sobre as relações interpessoais no cuidado de enfermagem ambulatorial. Métodos: Investigação descritiva de abordagem qualitativa, delineada sob a ótica da hermenêutica dialética. Participaram 12 enfermeiros atuantes em duas Unidades de Pronto Atendimento, tendo sido a entrevista estruturada realizada no mês de setembro de 2016, com duração de \pm 00:30:00 minutos. A análise de dados foi realizada segundo as etapas da hermenêutica dialética. Atenderam-se todos os aspectos ético-legais em pesquisa conforme legislação (inter)nacional. Resultados: Foram identificados três núcleos de sentido: relações interpessoais no trabalho em equipe multiprofissional de saúde; relações interpessoais $x$ cuidado de enfermagem; sentimentos dos usuários, julgamentos conflitantes e o cuidado de enfermagem. Conclusão: A cooperação como ferramenta das relações interpessoais pode contribuir para diminuir a insatisfação dos usuários, bem como limitar os conflitos da prática médica hegemônica que impacta negativamente o cuidado do enfermeiro ao usuário. Sugere-se a realização de novas investigações com enfoque intervencionista, a exemplo das atividades de educação permanente em saúde.

Palavras-chave: cuidados de enfermagem, assistência ambulatorial, relações interpessoais.

\section{Abstract \\ Nurses' perceptions of interpersonal relationships in outpatient nursing care}

Introduction: Interpersonal relationships, through the expansion of dialogue and the promotion of participatory management, constitute a fundamental competence in care and in strengthening humanization in outpatient care. Objective: To understand nurses' perceptions of interpersonal relationships in outpatient nursing care. Methods: Descriptive investigation of a qualitative approach outlined from the perspective of dialectical hermeneutics. Twelve nurses working at two Emergency Care Units participated, and the structured interview was conducted in September 2016 , lasting \pm 00:30:00 minutes. Data analysis performed according to the steps of dialectical 
hermeneutics. All ethical and legal aspects of research were complied with according to (inter) national legislation. Results: Three cores of meaning were identified: interpersonal relationships in multi professional health teamwork; interpersonal relationships $\mathrm{x}$ nursing care; users' feelings, conflicting judgments and nursing care. Conclusion: Cooperation as a tool for interpersonal relationships can contribute to reduce users 'dissatisfaction, as well as limit the conflicts of hegemonic medical practice that negatively impacts nurses' care for users. Further investigations with an interventionist approach are suggested, such as permanent health education activities. Keywords: nursing care, ambulatory care, interpersonal relations.

\section{Resumen \\ Percepciones de las enfermeras sobre las relaciones interpersonales en la atención de enfermería ambulatoria}

Introducción: Las relaciones interpersonales, a través de la ampliación del diálogo y la promoción de la gestión participativa, se configuran como competencia fundamental en el cuidado y fortalecimiento de la humanización en la atención ambulatoria. Objetivo: Comprender la percepción de las enfermeras sobre las relaciones interpersonales en la atención ambulatoria de enfermería. Metodología: Investigación descriptiva con enfoque cualitativo, planteada desde la perspectiva de la hermenéutica dialéctica. Participaron 12 enfermeros que laboraban en dos Unidades de Emergencias, y la entrevista estructurada se realizó en septiembre de 2016, con una duración de \pm 00:30:00 minutos. El análisis de los datos se realizó según los pasos de la hermenéutica dialéctica. Todos los aspectos éticos y legales de la investigación se cumplieron de acuerdo con la legislación (inter) nacional. Resultados: Se identificaron tres núcleos de significado: relaciones interpersonales en el trabajo en equipos multiprofesionales de salud; relaciones interpersonales $x$ cuidados de enfermería; sentimientos de los usuarios, juicios conflictivos y cuidados de enfermería. Conclusión: La cooperación como herramienta para las relaciones interpersonales puede contribuir a reducir la insatisfacción de los usuarios, así como limitar los conflictos de la práctica médica hegemónica que impacta negativamente en la atención de las enfermeras a los usuarios. Se sugiere que se realicen nuevas investigaciones con un enfoque intervencionista, como las actividades de educación permanente en salud.

Palabras-clave: atención de enfermería, atención ambulatoria, relaciones interpersonales.

Introdução

O enfermeiro não atua isoladamente, mas por meio de interações com outros profissionais de saúde para alcançar seus objetivos [1,2]. Destarte, a habilidade de saber relacionar-se com as pessoas é fundamental para a sua prática, pois pode contribuir para a humanização no ambiente de trabalho e melhoria da qualidade do atendimento às pessoas que são assistidas nos diferentes níveis de saúde [3,4].

A conservação de relacionamentos interpessoais saudáveis pode ser facilitada por diálogo aberto e claro, respeito e confiança entre os profissionais e entre estes e usuários. Além disso, as relações interpessoais, por meio da ampliação do diálogo e da promoção da gestão participativa, surgem como competência fundamental para o cuidado e para o fortalecimento da humanização na assistência ambulatorial [5,6].

O cuidado exige do profissional que aspira realizá-lo algumas qualidades: ética, solidariedade e confiança, uma vez que tem como objetivo o alívio, o conforto do ser cuidado, podendo promover a cura, o bem-estar e, quiçá, a mudança de estilo de vida deste $[1,2,7]$. $O$ cuidado humanizado está regulado no respeito à individualidade dos usuários e na dissociação de comportamentos sociais negativos, discriminação e medo $[3,8]$. Apesar de o Sistema Único de Saúde (SUS) e suas políticas focalizarem a tentativa de alcance do cuidado humanizado, a formação dos profissionais de saúde, em sua maioria, segue a lógica de um cuidado fragmentado, especializado, biologizante e focado no modelo liberal [5,7].

A assistência ambulatorial aqui se refere ao atendimento nas Unidades de Pronto Atendimentos (UPAs), que são estruturas de complexidade intermediária entre as Unidades Básicas de Saúde (UBSs) e a rede hospitalar, funcionando 24h por dia, todos os dias da semana. Ela é composta por uma rede organizada de atenção a urgências e emergências, com pactos e fluxos previamente definidos, com o objetivo de garantir o acolhimento aos usuários, intervir em sua condição clínica e contrarreferenciá-los para os demais pontos de atenção [5,9].

O enfermeiro é indicado pelo Ministério da Saúde (MS) como sendo o responsável pela avaliação inicial do usuário que chega à UPA e determina a prioridade de atendimento assim como o tempo de espera, organizando o fluxo de triagem e promovendo um cuidado humanizado. 
Ressalta-se que todos os profissionais de saúde devem realizar o acolhimento do usuário e sua família, mas cabe ao enfermeiro a atividade de classificação de risco [10,11].

Nessa perspectiva, o enfermeiro contribui para o SUS de forma diversificada ao contabilizar, em sua força de trabalho, mais de um milhão de trabalhadores, inseridos nos diferentes níveis de atenção à saúde no território brasileiro, com assistência ininterrupta, com estabelecimento de vínculo direto com os usuários dos serviços de saúde, equipe interdisciplinar e sociedade civil [2]. Isso justificou a realização deste estudo com enfoque na assistência ambulatorial visto suas peculiaridades referentes às características do ambiente terapêutico, às configurações de organização do trabalho e às relações interpessoais estabelecidas.

Foram elaboradas duas questões de pesquisa, a saber: Como os enfermeiros percebem as relações interpessoais durante a realização da assistência ambulatorial? Essas percepções são capazes de influenciar a qualidade do cuidado de enfermagem prestado? Sendo assim, as relações interpessoais no cuidado de enfermagem ambulatorial percebidas pelos enfermeiros foram consideradas objeto do presente estudo.

Desse modo, objetivou-se compreender as percepções de enfermeiros sobre as relações interpessoais no cuidado de enfermagem ambulatorial.

\section{Material e métodos}

Investigação de delineamento qualitativo de abordagem descritiva, fundamentada no método Hermenêutico-dialético como aporte à análise e tratamento dos dados [12,13], com base no protocolo Consolidated Criteria for Reporting Qualitative Research (COREQ) [14].

A pesquisa foi realizada em duas UPAs da cidade de Juiz de Fora, Minas Gerais, consideradas como referências macrorregionais no atendimento de urgência e emergência aos usuários do SUS. Cabe mencionar que, nos dois cenários de investigação, o enfermeiro é o responsável pela triagem, classificação de risco e acolhimento dos usuários.

Foram critérios de elegibilidade: os enfermeiros atuantes nas duas UPAs, cenários investigados $(\mathrm{N}=13)$. Foram critérios de exclusão: estarem em período de férias ou de licença médica, ou ainda se recusarem a participar ou adiarem a entrevista por $\geq 3$ abordagens. Houve apenas uma perda, um enfermeiro por recusa, totalizando-se 12 participantes inclusos na investigação.

O instrumento de coleta de dados, da entrevista semiestruturada, foi composto de: 1) Dados de caracterização sociodemográfica; 2) Questões norteadoras consideradas disparadoras sobre sua percepção individual a respeito das relações interpessoais relacionadas ao cuidado de enfermagem realizado no contexto ambulatorial da UPA e 3) Diário de campo. Cabe mencionar que o instrumento foi elaborado pelos pesquisadores após revisão prévia da literatura e levantamento dos pontos relevantes referentes às relações interpessoais no cuidado de enfermagem.

Após aquiescência, externada mediante a assinatura do Termo de Consentimento Livre e Esclarecido (TCLE) pós-informado, no horário agendado, as entrevistas ocorreram em sala com privacidade, evitando-se possíveis constrangimentos e proporcionando maior entrosamento da díade pesquisador-participante. O processo de coleta de dados foi antecedido por agendamento individual realizado pessoalmente, em horário de serviço pela pesquisadora principal, conforme disponibilidade do binômio pesquisador-participante. A coleta de dados ocorreu em setembro de 2016, com duração estimada de \pm 00:30:00 minutos.

Cabe mencionar que os dados de caracterização sociodemográfica foram registrados em impresso próprio, os provenientes das questões norteadoras tiveram seu conteúdo total gravado em áudio para posterior transcrição e as informações complementares relevantes, assim como as impressões da pesquisadora, obtidas durante a entrevista, foram registradas em diário de campo.

Os dados foram transcritos na íntegra em Software Word for Windows 2016 e elaboradas as categorias analíticas segundo o conteúdo [14], sendo o tratamento de dados conforme o método Hermenêutico-dialético [13]. Essa análise foi realizada seguindo as etapas: 1) Elaboração das categorias analíticas no início da pesquisa para o desvendamento dos aspectos mais abstratos e mediadores do contexto da pesquisa. Foram elas: o trabalho do enfermeiro na assistência ambulatorial; percebendo as relações interpessoais com o usuário na assistência ambulatorial; relações interpessoais como limitantes ao cuidado de enfermagem. 2) Organização dos dados, em que todas as entrevistas foram transcritas e, posteriormente, fez-se a leitura flutuante do material, o que possibilitou que a pesquisadora fosse impregnada pelo seu conteúdo. 3) Classificação dos dados - teve por objetivo a identificação dos núcleos de sentido a partir das 
falas dos entrevistados, denominadas aqui unidades de sentido. Os núcleos de sentido são estruturas de relevância que possibilitaram a interpretação e inter-relação das ideias centrais, bem como a identificação do que foi relatado com mais evidência pelos entrevistados, que são as unidades de sentido [12].

O número de entrevistados foi definido pelo adensamento teórico que foi identificado pela repetição e frequência com que emergiram os conteúdos categóricos e ausência de novas informações complementares a respeito do objeto investigado. Foi concebido que a amostra qualitativa foi ideal pela capacidade de refletir, em quantidade e intensidade, as múltiplas dimensões de determinado fenômeno, garantindo a qualidade e integração dos dados [15,16].

O projeto de pesquisa foi aprovado pelo Comitê de Ética em Pesquisa da Universidade Federal de Juiz de Fora (CEP-UFJF) da pesquisa intitulada "Relações interpessoais no acolhimento com usuários na classificação de risco: percepção do enfermeiro", sob o parecer consubstanciado oㅜ 1.698190 aprovado em 25/08/2016, e atendeu os aspectos ético-legais de pesquisa em seres humanos conforme legislação (inter)nacional. $O$ anonimato dos participantes foi garantido, mediante a adoção de códigos alfanuméricos compostos pela abreviatura da palavra enfermeiro "ENF" e dois dígitos que corresponderam à ordem de realização das entrevistas.

\section{Resultados}

Dos 12 enfermeiros entrevistados, sete eram do sexo feminino $(58,33 \%)$, com idade compreendida entre 27 e 48 anos, o tempo de formado variou entre três e 12 anos, e a atuação no contexto de urgência e emergência nas UPAs variou entre um e seis anos. Do total de participantes, nove trabalhavam como plantonistas em esquema $12 \times 36 \mathrm{~h}$ e três em regime diarista, dois $(16,66 \%)$ tinham mais de um vínculo empregatício, e apenas seis deles $(50 \%)$ possuíam alguma pós-graduação.

Os núcleos de sentidos que emergiram das falas foram: "Relações interpessoais no trabalho em equipe multiprofissional de saúde"; "Relações interpessoais como assistência ao usuário"; "O cuidado de enfermagem: sentimentos dos enfermeiros no acolhimento" que foram discutidos em forma de categorias analíticas.

O trabalho do enfermeiro na assistência ambulatorial - Núcleo de Sentido: Relações interpessoais no trabalho em equipe multiprofissional de saúde

Os participantes destacaram as relações interpessoais no trabalho da equipe multiprofissional de acordo com as unidades de sentido:

Então, eu tenho que ter um bom relacionamento. Se eu tenho esse bom relacionamento, eu ajudo eles e eles me ajudam [equipe de enfermagem]. E, com os médicos, é a mesma coisa. ENF06.

Eu tenho que me dar bem com o meu colega de trabalho. Fica um ambiente bom, tranquilo de trabalhar. ENF10.

[...] Então a relação interpessoal é muito importante. Porque eu saber lidar com o meu colega, né, o serviço dele adiciona e quem vai se sentir melhor é o paciente que vem procurar a unidade, né? ENF11.

Os participantes também destacaram que adequadas relações interpessoais impactam positivamente o cuidado de enfermagem ao usuário:

Eu acho que é aqui ele se sente acolhido. Não é só ele vem para ser medicado. Mas, na maioria das vezes, por trás de uma simples dor de cabeça, às vezes, tem um problema familiar, entendeu? Então assim, o simples fato dele ver que está todo mundo junto ele já se sente acolhido. ENF01.

Ele está com uma queixa, aí a gente conversando, descobre que o problema dele não é a dor de cabeça, porque ele está com uma pressão alta. É alguma coisa que aconteceu com ele e está gerando essa dor de cabeça. ENF03.

Positivamente. Porque eles acreditam muito na gente. A nossa palavra, para eles, vale muito. ENF04. 
Percebendo as relações interpessoais no cuidado de enfermagem - Núcleo de Sentido: Relações interpessoais $x$ cuidado de enfermagem

Os participantes percebem que a interação com os usuários é necessária para que o cuidado de enfermagem se realize efetivamente, como observado nas unidades de sentido.

Alguns vêm fragilizados, que choram, que contam a sua vida ali. Em poucos minutos, você fica conhecendo o paciente. Mas tem outros que, se você não perguntar, eles não soltam, são mais retraídos, entendeu? Principalmente homens, idosos (risos). "Minha filha, não gosto de vir em médico". Aí, se você não perguntar, não cutucar, não libera. ENF05.

Ah, passa um relacionamento do profissional com o paciente. Que é um relacionamento, assim, de acolher mesmo, de saber o que está se passando, de tratamento, de cuidado [...] isso reflete até nas pessoas, na hora da triagem em si. ENF12.

Além disso, os enfermeiros percebem que as relações interpessoais emergem da escuta qualificada, que é um elemento essencial na assistência ambulatorial como descrevem as unidades de sentido.

Normalmente, modéstia à parte, eu venho recebendo alguns elogios da maneira pela qual eu trabalho. Por quê? Eu escuto o paciente. ENF04.

É a conversa que você tem no dia a dia. É paciente que a gente já pega até uma amizade. ENF05.

$E$, muitas das vezes, não é o atendimento imediato que você precisa prestar, mas é mais a escuta, é uma dor que já é crônica, você precisa mesmo de acolher mesmo, do que de propriamente assistir imediatamente. ENF07.

As relações interpessoais e o limite ao cuidado de enfermagem - Núcleo de Sentido: Sentimentos dos usuários, julgamentos conflitantes e o cuidado de enfermagem

Os participantes relatam que os sentimentos dos usuários limitam o cuidado de enfermagem como citado nas unidades de sentido a seguir:

Bom, para mim, a dificuldade é essa questão de cultura mesmo. Enquanto uns que entendem [...]. Igual tinha um ali, um já trabalhava na saúde e a outra não. A outra que é mais ligada à filha. Ela entendeu um pouco o nosso lado, que nem tudo é a gente que resolve. A gente depende também de outras pessoas para ajudar. ENF02.

$E$, eu acho que eles não gostam muito da triagem. Sentem um pouco de resistência. "Ainda tem que passar pela triagem?" Aí, às vezes, a gente faz a classificação: "Por que que a minha classificação é verde? É urgência o meu atendimento". ENF03.

[...] Os pacientes acabam ficando um pouco agressivos com a gente que está na triagem. Então, às vezes, acaba indispondo com a gente por uma coisa que não é nem culpa nossa. É questão médica (médicos demorando a atender). ENF07.

Os participantes relatam que outras categorias profissionais avaliam de forma equivocada o cuidado de enfermagem na assistência ambulatorial:

[...] existem alguns médicos que não curtem muito essa coisa da triagem do enfermeiro. Mas o médico ele acha que ele tem o direito de colocar o dedo na sua triagem ou de falar que a sua triagem não está certa e tal. Isso já aconteceu comigo e já aconteceu com vários outros funcionários. Eu acho isso um problema. ENF05.

Eu acho que precisa, aí sim, do relacionamento interpessoal, mais afetivo com o médico. Ele prefere questionar a triagem a pegar o paciente ou refazer a triagem. Ou assim, eu acho que não existe uma retriagem para menos, né? Só para mais. Mas, às vezes, o médico acha que o paciente está triado de uma coisa que é menos e não valoriza a nossa triagem. Eu acho que o que prejudica é o relacionamento interpessoal com o médico. ENF08. 
Um perfil similar de caracterização sociodemográfica e profissional foi encontrado em outra investigação realizada com enfermeiros da Atenção Básica [17]. Entre os inúmeros espaços em que ocorrem cuidados de enfermagem, os serviços de assistência ambulatorial constituem-se em um dos segmentos da saúde em que os enfermeiros exercem sua prática como integrantes da equipe multiprofissional e interdisciplinar [18].

Embora haja alinhamento teórico entre a equipe multiprofissional com os fundamentos da assistência ambulatorial, é necessário ampliar a sua concepção para além de uma atividade específica, exigindo novos investimentos sobre a habilidade do cuidar e do estar atento para acolher, o que potencializa o atendimento das demandas de cuidados e necessidades básicas dos usuários [19]. Isso é condição para favorecer o cuidado humanizado na atuação do enfermeiro na assistência ambulatorial, uma vez que parte dessas habilidades são as boas relações interpessoais na equipe multiprofissional, o bem-estar do enfermeiro, o trabalho reconhecido e valorizado, a formação e a capacitação [20].

As unidades de sentido demonstraram que boas relações interpessoais com os demais profissionais da equipe de saúde viabilizam a criação de um ambiente de colaboração tranquilo e fazem com que o usuário se sinta bem. Além disso, relações interpessoais adequadas com os usuários são positivas ao aumentarem a confiança destes com relação ao enfermeiro, resultando, por exemplo, em uma anamnese mais abrangente [18-20].

Pode-se inferir que o trabalho do enfermeiro na assistência ambulatorial, sob a perspectiva das relações interpessoais, não é uma prática simples, uma vez que depende de múltiplos fatores: sociais, históricos, culturais, econômicos, enfim, exige uma equipe multiprofissional que atue de forma interdisciplinar e tenha uma formação adequada e educação permanente em saúde, além de condições laborais e de trabalho reconhecido e valorizado [1,2].

A percepção é um fator de ordem psicológica, como a aprendizagem, as crenças e as atitudes. O enfermeiro, por vezes, não consegue perceber tudo relacionado ao seu processo de trabalho, ele usa a exposição seletiva, isto é, interpreta a mensagem e a adapta aos seus valores para decidir quais estímulos irá notar e quais irá ignorar. Ao decidir, esta ação perpassa por influências conscientes, inconscientes, intencionais ou não. Destarte, um comportamento pode ser induzido pela influência, que habitualmente ocorre nas relações interpessoais [21].

No que concerne ao cuidado de enfermagem no ambulatório, observa-se que ele é marcado por ambiguidades e por relações interpessoais conflitantes, que podem culminar em vivências de angústia e de sofrimento, bem como em um cuidado de enfermagem inadequado [22]. Destaca-se que a percepção do enfermeiro abrange um processo pelo qual ele seleciona, organiza e interpreta estímulos, traduzindo-os em uma imagem significativa e coerente para 0 cuidado a ser prestado $[2,21]$.

A manutenção de relações interpessoais saudáveis no cuidado de enfermagem é possibilitada por um diálogo franco e transparente, respeito e confiança entre enfermeiro e usuário. Este se constitui num instrumento ímpar para alcançar melhorias na assistência oferecida ao indivíduo e, consequentemente, atingir resultados terapêuticos bem-sucedidos [6].

Neste estudo, o cuidado de enfermagem foi o enfoque, no entanto cabe destacar que 0 cuidado é uma função que deve perpassar todas as categorias profissionais inseridas no âmbito da saúde. Todos os membros da equipe interdisciplinar de saúde devem ter o cuidado em suas práxis, considerando que essa é uma área que está sujeita a condições políticas, econômicas, ideológicas e tecnológicas. Esse cuidado exige ética, solidariedade e confiança, tendo como objetivo o alívio, o conforto, podendo promover a cura, o bem-estar e melhorias nos hábitos de vida dos usuários [2,7].

Com base nos resultados apresentados, observa-se que a interação entre enfermeiros e usuários é necessária para que o cuidado de enfermagem se realize, pois alguns destes últimos relatam sua vida em poucos minutos. Entretanto, a interação com outros usuários, ao contrário, exige que o enfermeiro tenha uma maior habilidade para tanto. A escuta qualificada deve ser considerada como elemento essencial na assistência ambulatorial [1], conforme evidenciado no discurso dos participantes.

Nessa perspectiva, a percepção do enfermeiro, as relações interpessoais entre o enfermeiro e o usuário e o cuidado de enfermagem estão intimamente relacionados e são, por conseguinte, indissociáveis da equipe interdisciplinar. Destaca-se que o enfermeiro, mesmo que de forma incipiente, percebe que o cuidado de enfermagem está articulado com as peculiaridades da assistência ambulatorial [5]. 
Os sentimentos negativos existentes na assistência ambulatorial têm origem na diferença que se reflete entre cuidado e intervenção de enfermagem. Intervir aqui assume o significado de fazer algo para o usuário, desconsiderando a subjetividade da interação enfermeiro-usuário. Por conseguinte, o cuidado de enfermagem deve ressaltar a subjetividade dessa interação, valorizando-a na interpretação do processo saúde-doença do usuário [7].

$\mathrm{Na}$ atualidade, os julgamentos conflitantes perpassam pelo modelo biomédico ainda engessado, que corrobora a construção de uma interpretação do cuidado de enfermagem como uma práxis fragilizada. Essa interpretação culmina em concepções do senso comum, por vezes percebida nos próprios profissionais de enfermagem e nas relações interpessoais constituintes da assistência ambulatorial [23].

Os participantes relataram que alguns usuários, devido a características culturais, por desconhecimento, apresentam resistência com relação à assistência ambulatorial oferecida, outros, "mais esclarecidos", compreendem o processo. Cabe mencionar ainda a exteriorização de uma insatisfação quanto à conduta de alguns profissionais da classe médica em relação à percepção de um julgamento sobre o cuidado de enfermagem de forma depreciativa e, por vezes, equivocada e desrespeitosa.

Desse modo, vale destacar que sentimentos negativos de usuários, bem como julgamentos conflitantes com relação ao cuidado de enfermagem, trazem prejuízos não só à categoria de enfermagem, mas também às relações interpessoais da equipe multiprofissional e ao usuário. Além disso, repercute no sistema como um todo, aumentando o tempo de atendimento e os custos dos serviços com o retrabalho. Enfim, isso pode gerar um círculo vicioso nocivo para a assistência ambulatorial [1,2].

Conclusão

Ao compreender as percepções de enfermeiros sobre as relações interpessoais no cuidado de enfermagem ambulatorial, evidenciou-se que estes reconhecem ferramentas essenciais a essas relações, a exemplo de humanização, ética, acolhimento, escuta qualificada, e atuação interdisciplinar respeitosa. A cooperação deve ocorrer como ferramenta das relações interpessoais e pode contribuir para diminuir a insatisfação dos usuários, bem como limitar os conflitos da prática médica hegemônica que impacta negativamente o cuidado do enfermeiro ao usuário.

Como contribuições desta investigação foram apresentadas percepções dos profissionais de enfermagem a respeito do cuidado ambulatorial e de suas relações interpessoais na tríade enfermagem, equipe interdisciplinar e usuários de modo a proporcionar reflexões sobre a assistência de enfermagem no contexto ambulatorial para o planejamento de ações educativas que visem à otimização do cuidado humanizado e holístico, tendo como base a melhoria das relações interdisciplinares.

Foram considerados limites desta investigação o número reduzido de participantes, que foi justificado pela abordagem apenas de enfermeiros, e a não inclusão de todas as UPAs pertencentes à cidade investigada. É recomendação dos autores a realização de novas investigações sobre a temática, que abordem todos as UPAs da região e novos delineamentos de investigação, a exemplo do método quantitativo e misto.

\section{Referências}

1. Eloia SMC, Vieira RM, Eloia SC. The interpersonal relationship between professionals of the Family Health Strategy. Essentia-Revista de Cultura, Ciência e Tecnologia da UVA 2019;20(1):1-8. https://doi.org/10.36977/ercct.v20i1.249

2. Rocha EM, Lucena AF. Single therapeutic project and nursing process from an interdisciplinary care perspective. RGE 2018;39:e2017-0057. https://doi.org/10.1590/1983-1447.2018.2017-0057

3. Brasil. Política Nacional de Humanização. 1르 ed. Brasília: Ministério da Saúde; 2013. p.16.

4. Brasil. Distrito Federal (DF). Secretaria de Estado da Saúde (SES). Portaria ํo 773 , de 19 de julho de 2018. Estabelece diretrizes e normas para a organização da Atenção Ambulatorial Secundária. DO do DF 2018. Brasília: Diário Oficial; 2018.

5. Brasil. Conselho Nacional de Secretários de Saúde (CONASS). Planificação da Atenção à Saúde - uma proposta de gestão e organização da Atenção Primária à 
Saúde e da Atenção Ambulatorial Especializada nas Redes de Atenção à Saúde. Brasília: Conass; 2019.

6. Peruzzo HE, Bega AG, Lopes APAT, Haddad MCFL, Peres AM, Marcon SS. The Challenges of teamwork in the Family Health Strategy. EAN 2018;22(4):e20170372. https://doi.org/10.1590/2177-9465-ean-2017-0372

7. Carnut L. Care, integrality and primary care: essential articulation to reflect on the health sector in Brazil. Saúde Debate 2017;41(115):1177-86. https://doi.org/10.1590/0103-1104201711515

8. Santos BM, Silva RMCRA, Pereira ER, Joaquim FL, Goés TRP. Nursing student's perception about humanized care: integrative review. REBEN 2018;71(suppl6):2800-7. https://doi.org/10.1590/0034-7167-2017-0845

9. Brasil. Manual instrutivo da Rede de Atenção às Urgências e Emergências no Sistema Único de Saúde (SUS). Brasília: Ministério da Saúde; 2013.

10. Campos TS, Arboit ÉL, Mistura C, Thum C, Arboit J, Camponogara S. User embracement and risk classification: health professionals' and users' perceptions. Revista Brasileira em Promoção da Saúde 2020;33:9786. https://doi.org/10.5020/18061230.2020.9786

11. Hermida PMV, Nascimento ERP, Echevarriá-Guanilo ME, Brüggemann OM, Malfussi LBH. User embracement with risk classification in an emergency care unit: an evaluative study. Rev Esc Enferm USP 2018;52:e03318. https://doi.org/10.1590/s1980220x2017001303318

12. Stein E. Dialética e Hermenêutica: uma controvérsia sobre método e filosofia. In: Dialética e Hermenêutica. (Jurgen Habermas). São Paulo: L\&PM; 1987. p.98-134.

13. Minayo MCS. O desafio do conhecimento: pesquisa qualitativa em saúde. 14. ed. São Paulo: Hucitec; 2014.

14. Tong A, Sainsbury $P$, Craig J. Consolidated criteria for reporting qualitative research (COREQ): a 32-item checklist for interviews and focus groups. Int J Qual Health Care 2007;19(6):349-57. https://doi.org/10.1093/intghc/mzm042

15. Minayo MCS. Sampling and saturation in qualitative research: consensuses and controversies. Revista Pesquisa Qualitativa 2017;5(7):1-12. Disponível em: https://editora.sepq.org.br/index.php/rpq/article/view/82

16. Arreguy-Sena C, Melo LD, Braga LM, Krempser P, Lemos RDCPB, Lopes DP. Peripheral vein puncture in hospitalized adults: nested sequential mixed method. Enferm Bras 2019;18(6):775-83. https://doi.org/10.33233/eb.v18i6.3255

17. Pereira APN, Arreguy-Sena C, Queiroz ABA, Dutra HS, Melo LD, Krempser P. Social representations of primary care nurses on nursing records. Enferm Bras 2019;18(6):759-66. https://doi.org/10.33233/eb.v18i6.3219

18. Filho LAM, Martini JG, Lazzari DD, Vargas MAO, Backes VMS, Farias GM. Urgency/emergency course content in the education of generalist nurses. REME 2017;21:e-1070. https://doi.org/10.5935/1415-2762.20170016

19. Faria MC, Vargas CRM. User embracement: conceptions and practices of the professionals who are in interdisciplinar services at Hospital Universitário de Brasília. Tempus 2017;11(3):163-75. https://doi.org/10.18569/tempus.v11i3.2472

20. Rodrigues AC, Calegari T. Assistance humanization in pediatric intensive care unit: perspective of nursing staff. REME 2016;20:e933. https://doi.org/10.593/14152762.20160003

21. Endo ACB, Roque MAB. Attention, memory and perception: a conceptual analysis of neuropsychology applied to advertising and its influence on consumer behavior. Intercom - RBCC 2017;40(1):77-95. https://doi.org/10.1590/1809-5844201715

22. Caram CS, Resende LC, Brito MJM. Colaborative practice: potentialities and challenges for nurses in the Hospital Contexto. REME 2017;21:e1070. https://doi.org/10.5935/1415-2762.20170080

23. Oliveira CN, Nunes EDCA. Caring for family members in the ICU: Challenges faced by nurses in the interpersonal praxis of user embracement. Texto Contexto Enferm 2014;23(4):954-63. https://doi.org/10.1590/0104-07072014003590013 